\title{
Contribuições das práticas alimentares e inatividade física para o excesso de peso infantil
}

\author{
Feeding practices and physical inactivity contributions to childhood overweight
}

\author{
Ana Elisa M. Rinaldi ${ }^{1}$, Avany Fernandes Pereira ${ }^{2}$, Célia Sperandeo Macedo ${ }^{3}$, João Felipe Mota ${ }^{4}$, Roberto Carlos Burini ${ }^{5}$
}

\section{RESUMO}

Objetivo: Revisar estudos que abordam as práticas alimentares atuais e o padrão de atividade física como contribuintes do excesso de peso na infância.

Fontes de dados: Ovid Journals, Highwire e SciELO, com seleção de artigos originais e de revisão nos últimos dez anos (1997 a 2007), na língua portuguesa e inglesa.

Síntese de dados: O acompanhamento do estado nutricional de crianças permite diagnosticar seu estado de saúde atual, bem como predizer parcialmente seu prognóstico na vida adulta. A prevalência de obesidade infantil, no Brasil, apresenta aumento progressivo em todas as classes sociais e sua freqüência varia entre cinco a $18 \%$, dependendo da região estudada. A associação da transição epidemiológica, demográfica e comportamental e a alteração do hábito alimentar são apontadas como fatores causais do aumento progressivo da obesidade infantil. Práticas alimentares caracterizadas por elevado teor de lipídios, sacarose e sódio e por reduzido consumo de cereais integrais, frutas e hortaliças associadas à inatividade física decorrente do uso de computadores, jogos eletrônicos e televisores influenciam parte considerável de crianças. Este estilo de vida reflete os hábitos familiares e pode ser influenciado pelo ambiente escolar no qual a criança está inserida.

Conclusões: Os dados sugerem influência considerável dos fatores ambientais, principalmente hábitos alimentares e inatividade física, no crescente aumento da prevalência de excesso de peso na população pediátrica.

Palavras-chaves: obesidade; transição nutricional; consumo alimentar; atividade física.

\section{ABSTRACT}

Objective: To review the literature in order to show how current feeding and physical activity practices may contribute to childhood overweight.

Data source: Ovid Journals, Highwire and SciELO, selecting original and review articles from 1997 to 2007, published in English and Portuguese.

Data synthesis: The periodic assessment of children nutritional status is important to diagnose their current health status and to predict their adult life prognosis. In Brazil, the prevalence of childhood obesity is progressively increasing in all social classes and its frequency varies from five to $18 \%$, according to the region assessed. The association between the health, demographic and behavioral transition and the change in feeding practices can explain the increasing prevalence of childhood overweight. The current food consumption with high fat, sugar and sodium intake and low intake of whole cereals, fruits and vegetables associated to physical inactivity due to the excessive use of computers, electronic games and television may play a role in childhood obesity. This life style can be explained by changing family

Endereço para correspondência

Ana Elisa Madalena Rinaldi

Rua Visconde do Rio Branco, 898 - apto. 10

CEP 18602-000 - Botucatu/SP

E-mail: anarinaldi@usp.br

Recebido em: 12/2/2008

Aprovado em: 2/6/2008 
habits and school environment.

Conclusions: These data suggest considerable influence of environmental factors, mainly nutritional habits and physical inactivity, on the increasing prevalence of childhood overweight.

Key-words: obesity; nutritional transition; food consumption; physical activity.

\section{Introdução}

Nas eras paleolítica e neolítica, o homem primitivo se desenvolveu em um ambiente em que os alimentos eram de fontes naturais, o abastecimento incerto e as restrições sazonais de consumo eram freqüentes. Deste modo, o homem evoluiu com reservas nos momentos de fartura e mecanismos adaptativos que o auxiliavam a sobreviver durante os períodos de escassez ${ }^{(1)}$.

Esta grande capacidade de armazenar energia do alimento pode ser considerada uma característica genética negativa para o homem contemporâneo, pois o desenvolvimento tecnológico e a mecanização da produção favoreceram o aumento exponencial da disponibilidade de alimentos e, paralelamente, o gasto energético com as atividades laborais e de lazer diminuiu de forma significativa ${ }^{(2)}$. Neste sentido, a carga genética ancestral ainda se expressa na forma de deposição do excesso energético, manifestando-se por hiperadiposidade. Desse modo, na segunda metade do século passado, a hiperadiposidade corporal, que a paleoantropometria considerava sinal de sobrevivência e fertilidade, bem como saúde, beleza, status social e riqueza, surgiu como fator de risco para o desenvolvimento de doenças crônicas não transmissíveis como diabetes melito tipo 2 (DM2), hipertensão arterial sistêmica (HAS), dislipidemias, doenças reumáticas e alguns tipos de neoplasias ${ }^{(3)}$.

Nos últimos 20 anos, a população pediátrica também se tornou alvo deste excesso de gordura corporal, possivelmente devido a hábitos alimentares inadequados e à inatividade física.

O estilo de vida atual, resultante de um conjunto de fatores como inserção da mulher no mercado de trabalho, violência nas grandes cidades, aumento da carga horária escolar, refeições fora do domicílio, ausência dos pais nas refeições, entre outros, pode contribuir para a instalação da obesidade, com repercussões na saúde infantil e na vida adulta ${ }^{(4-6)}$.

A obesidade é preocupante não apenas pelas implicações à saúde, como pela complexidade de seu tratamento e controle, já que este acarreta em mudança de comportamento alimentar no plano individual e da adoção de políticas pú- blicas que podem ir contra os interesses de diferentes setores da indústria e comércio de alimentos ${ }^{(3)}$.

O presente trabalho tem como objetivo revisar estudos que abordam as práticas alimentares atuais e o padrão de atividade física como contribuintes para o excesso de peso na infância e foi dividido em quatro tópicos, que compreendem a revisão dos dados, além das conclusões.

Os tópicos abordam a prevalência de excesso de peso na população pediátrica brasileira, definição de transição nutricional com enfoque no Brasil, alterações do hábito alimentar e transição comportamental. Os artigos foram selecionados nas bases de dados do Ovid Journals, Highwire e SciELO, utilizando as palavras obesidade, transição nutricional, consumo alimentar e inatividade física. Foram selecionados os artigos publicados nos anos de 1997 a 2007, na língua portuguesa e inglesa. Procurou-se incluir artigos originais, de revisão, metanálise e artigos nacionais, por representarem o hábito da população brasileira.

\section{Diferenças regionais na prevalência de excesso de peso na população pediátrica brasileira}

A avaliação da evolução estatural é considerada um bom parâmetro para verificar as condições de vida do indivíduo e também da população. A estatura na maturidade é resultante da interação entre a herança genética, condições ambientais, estado nutricional e saúde na infância ${ }^{(7,8)}$. O consumo alimentar insuficiente, considerado estresse nutricional, acarreta atraso no crescimento esquelético e na estatura ${ }^{(9)}$.

Barker ${ }^{(10)}$ propôs que qualquer agravo nutricional, fundamentalmente a desnutrição, ocorrido em período crítico do crescimento e desenvolvimento, poderia ter efeito deletério durante toda a vida por induzir mecanismos adaptativos que, na vida adulta, favoreceriam o ganho de peso excessivo. Esta hipótese pode fundamentar em parte os achados da prevalência de desnutrição e obesidade no país.

O estado nutricional também poderia determinar a evolução econômica de um país, já que o índice de desnutrição contribuiria para a pobreza e o bom estado nutricional favoreceria a produtividade e desenvolvimento econômico ao permitir adequado trabalho físico, desenvolvimento cognitivo, rendimento escolar satisfatório e menor freqüência de doenças ${ }^{(11)}$.

No início da década de 1980, outro problema nutricional começou a ser diagnosticado com maior freqüência: a obesidade infantil. Esta era mais prevalente em países desenvolvidos e principalmente na população com menor poder 
aquisitivo, entretanto, passou a ser detectado no Brasil entre crianças de classes socioeconômicas mais altas, mudando este perfil nos anos mais recentes ${ }^{(7,12,13)}$.

A última Pesquisa de Orçamento Familiar (POF) verificou redução da desnutrição infantil tanto na região Norte quanto no Sudeste, porém mais pronunciada na primeira região, mostrando menor influência das desigualdades econômicas no estado nutricional. A prevalência de excesso de peso na população pediátrica, nos últimos 30 anos, aumentou em todas as regiões brasileiras e em todos os extratos de renda ${ }^{(14)}$. Dados do Instituto Brasileiro de Geografia e Estatística (IBGE) mostraram excesso de peso em 16,7\% dos adolescentes, sendo maior (22\%) no início da adolescência e declinando no final da referida faixa etária, com prevalência nas regiões Nordeste e Sul de 11,7 e 19,8\%, respectivamente ${ }^{(14)}$.

No Rio de Janeiro, estudo de crianças e adolescentes (quatro a 17 anos) de escolas municipais detectou sobrepeso em $18 \%$ das meninas e em $15 \%$ dos meninos ${ }^{(15)}$, enquanto que estudo em Brasília, com 452 escolares na faixa etária de seis a dez anos de escola privada, mostrou índices de 16,8\% de sobrepeso e 5,3\% de obesidade ${ }^{(16)}$. Investigação de grande amostra populacional, na cidade de Santos, São Paulo, com 10.822 crianças de sete a dez anos, detectou prevalência de $15,7 \%$ de sobrepeso e $18 \%$ de obesidade ${ }^{(12)}$. Na região Sul, a prevalência de sobrepeso em crianças de sete a dez anos variou de 17,9 a $19 \%$ e a obesidade atingiu até $14 \%$ das crianças avaliadas ${ }^{(17,18)}$.

No Norte do país há poucos estudos sobre excesso de peso em crianças, porém, há o trabalho de Capelli e Koifman ${ }^{(19)}$, os quais desenvolveram pesquisa em uma comunidade indígena, na qual encontraram prevalência de sobrepeso em $6,7 \%$ de crianças menores de dez anos. Na região Nordeste, em estudo realizado com 1.616 crianças e adolescentes, observou-se $14,5 \%$ de sobrepeso e $8,3 \%$ de obesidade, com maior prevalência de excesso de peso nos indivíduos com melhor condição econômica ${ }^{(20)}$.

Esta variabilidade na prevalência de sobrepeso e obesidade é explicada pelas diferenças regionais, período de coleta de dados e, principalmente, pela metodologia aplicada ${ }^{(21)}$.

A obesidade infantil pode estar relacionada ao aparecimento de comorbidades como HAS, dislipidemias e diabetes melito tipo $2^{(22)}$, com repercussões na vida adulta. $\mathrm{O}$ risco para o desenvolvimento de doenças cardiovasculares na vida adulta aumentou proporcionalmente ao aumento do índice de massa corpórea (IMC) na infância, em estudo de crianças dinamarquesas durante 46 anos $^{(23)}$.

\section{Transição nutricional}

O Brasil passou por mudanças estruturais nos últimos 50 anos, com aumento da expectativa de vida, redução da mortalidade infantil, diminuição da taxa de fecundidade e aumento da população urbana ${ }^{(9)}$. Tais modificações entram no conceito da transição demográfica, definida por Frederiksen ${ }^{(24)}$ como modificações no desenvolvimento da sociedade que provocam mudanças nos padrões de morbimortalidade. Esta, por sua vez, integra-se à transição epidemiológica caracterizada pela redução da mortalidade por doenças infecciosas e aumento das doenças crônicas não transmissíveis ${ }^{(25)}$. Integrada a estes conceitos está a transição nutricional, que se refere à mudança do estado nutricional da população, resultante, em parte, de modificações do estilo de vida, alteração do padrão dietético, inatividade física e determinada pela inter-relação dos fatores econômicos, demográficos e culturais ocorridos na sociedade, principalmente nas quatro últimas décadas do século $20^{(26)}$.

A transição nutricional pode ser dividida em cinco padrões distintos: o primeiro corresponde ao homem paleolítico (catador/caçador) que tinha uma dieta saudável (baseada em hortaliças e carnes magras), porém com baixa expectativa de vida devido aos traumas e doenças infecciosas. $\mathrm{O}$ segundo se refere ao período da agricultura (alto consumo de cereais e monotonia alimentar) e a emergente fome, com piora do estado nutricional. No terceiro padrão, a fome é superada, observando-se melhora da qualidade dietética (maior quantidade de frutas, hortaliças e proteína animal) e aumento da expectativa de vida. No quarto padrão, houve rápida mudança no consumo alimentar (elevada ingestão de gordura saturada, açúcar, alimentos processados e menor teor de fibras) e redução da atividade física, com maior risco para o desenvolvimento das doenças crônicas não transmissíveis. Por fim, o quinto padrão, no qual se pode notar princípios de mudanças positivas como maior do consumo de gordura monoinsaturada e fibras ${ }^{(27)}$.

No Brasil, Batista Filho e Rissin ${ }^{(9)}$ propuseram caracterizar a transição nutricional em quatro etapas. A primeira seria o desaparecimento da desnutrição edematosa (kwashiorkor), com alta mortalidade, seguida pela redução do marasmo (segunda etapa) e sua associação com doenças infecciosas. $\mathrm{Na}$ terceira etapa, pôde-se observar o aparecimento do binômio sobrepeso/obesidade em escala populacional e, por fim, a última etapa, definida pela correção do deficit estatural.

$\mathrm{Na}$ população pediátrica, a transição nutricional pode ser identificada em fases distintas em duas regiões brasileiras: 
no Norte e Nordeste, encontra-se no estágio inicial, com predominância da redução da baixa estatura e aumento da obesidade em lactentes, devido ao desmame precoce e aos erros alimentares nos primeiros anos de vida; no Sul e Sudeste, a transição nutricional estaria em estágio mais avançado, caracterizado pela redução da obesidade em crianças das zonas urbanas, devido à maior escolaridade das mães ${ }^{(28)}$.

\section{Alterações no consumo alimentar}

O consumo alimentar da criança brasileira ainda é pouco estudado e os dados do Estudo Nacional sobre Despesa Familiar (Endef) ${ }^{(29)}$, representativos da população, datam de $1974 / 75$.

Alguns autores têm mostrado a importância do consumo de frutas e hortaliças para prevenir a obesidade e suas comorbidades, porém a ingestão destes alimentos em crianças está bem abaixo dos valores recomendados ${ }^{(30,31)}$. Geralmente, alimentos com baixa palatabilidade como vegetais são oferecidos às crianças em contexto negativo pelos próprios pais ou responsáveis, dificultando a sua ingestão ${ }^{(32)}$.

Pela pirâmide alimentar brasileira ${ }^{(33)}$, recomenda-se para crianças em idade escolar seis porções de pães e cereais, quatro porções diárias de frutas e de hortaliças, uma porção de leguminosas, duas de carnes e ovos, três de leite e produtos lácteos, duas de açúcar e doces e uma de óleo e gordura. Para adolescentes as mesmas porções de frutas, leite e carnes, mas sete de cereais, quatro porções e meia de hortaliças, duas de leguminosas e uma porção meia de açúcar e óleo.

Ao analisar os estudos citados anteriormente, destacam-se como principais desvios alimentares: consumo insuficiente de frutas, hortaliças e leguminosas (principalmente feijão); ausência de refeições, com destaque para o desjejum; redução do consumo de leite e derivados com substituições dos mesmos por bebidas lácteas com menor concentração de cálcio; aumento no consumo de alimentos prontos (congelados e pré-preparados) e refrigerantes. Estes hábitos sinalizam para a necessidade de trabalho de educação alimentar envolvendo o núcleo familiar, os órgãos governamentais e os meios de comunicação ${ }^{(30-32,34)}$.

A relação entre práticas alimentares inadequadas e o excesso de peso é evidenciada por Mondini et al ${ }^{(31)}$, que verificaram maior razão de prevalência $(\mathrm{rp}=1,79, \mathrm{p}<0,05)$ para obesidade em crianças que consumiam quantidade superior de alimentos industrializados, tais como salgados fritos, batata frita, sanduíches, salgadinhos comercializados em pacotes, bolachas, balas e refrigerantes.
Resultados semelhantes são compartilhados por Triches e Giugliani ${ }^{(30)}$, que observaram, nas crianças com práticas alimentares menos saudáveis (não consumir frutas, hortaliças, leite, café-da-manhã e maior consumo de refrigerantes), chance cinco vezes mais elevada de serem obesas $(\mathrm{OR}=5,3 ; \mathrm{p}<0,05)$.

As escolas, ao oferecerem alimentos hipercalóricos nas cantinas, contribuem para o seu consumo inadequado. Crianças em creche consumiram maiores quantidades de leguminosas, hortaliças e frutas do que em casa ${ }^{(34)}$. No âmbito escolar, torna-se, portanto, necessário estimular o consumo de alimentos adequados por meio da oferta menos freqüente daqueles de alta densidade energética. Determinados componentes dos alimentos industrializados como gordura vegetal hidrogenada, sódio, açúcar em excesso são alvos de estudos que relacionam o seu consumo excessivo com o aumento da obesidade e comorbidades associadas ${ }^{(35-37)}$.

A gordura vegetal hidrogenada é a principal fonte de ácidos graxos trans, sendo encontrada em margarinas, cremes vegetais, pães, biscoitos, massas, bolos, sorvetes, batatas fritas e alimentos congelados. Apesar da recomendação da Organização Mundial da Saúde (OMS) de que o consumo máximo de gordura trans não ultrapasse $1 \%$ do valor energético total diário, na dieta ocidental estes valores representam 2,6\% ${ }^{(38)}$. A ingestão elevada deste tipo de lipídio aumenta as concentrações das lipoproteínas plasmáticas de baixa densidade e reduz as lipoproteínas de alta densidade ${ }^{(39)}$. No Brasil, não há estudos de avaliação da quantidade deste tipo de gordura consumida, mas a população compra elevadas quantidades de alimentos industrializados, sendo possível que sua ingestão seja elevada ${ }^{(40)}$. Atualmente os rótulos exibem a quantidade de gordura trans presente nos alimentos, facilitando sua identificação e análise.

O consumo de refrigerantes, constituído basicamente de açúcar ou edulcorantes na versão light/diet, está associado à redução da ingestão de leite e suco de frutas naturais ${ }^{(41)}$. Outro ingrediente utilizado como adoçante de bebidas e frutas industrializadas é o xarope de milho com alto teor de frutose. A razão para seu amplo uso é que a frutose apresenta maior solubilidade em meio aquoso, tem preço reduzido em até $70 \%$ e sua eficiência como adoçante é 1,7 vezes maior que a sacarose ${ }^{(42)}$.

O consumo excessivo de sal, superior a cinco gramas ao dia, é apontado como componente integrante da transição nutricional. A maior parte do sódio consumido é proveniente de produtos industrializados e, também, de alimentos preparados em restaurantes. Estudos populacionais relacio- 
nam o elevado consumo de sódio e o aumento da pressão arterial, enquanto a redução deste nutriente pode diminuir o risco de doenças cardiovasculares ${ }^{(43)}$. A elevada ingestão de sódio pode também reduzir a densidade mineral óssea por gerar carga ácida e estimular a excreção urinária de cálcio ${ }^{(37)}$.

Outro fator que merece destaque no contexto das práticas alimentares infantis é a duração do aleitamento materno e o momento da introdução dos alimentos de transição. Estudos apontam a importância do aleitamento materno no crescimento infantil e mostram a necessidade desta prática ser exclusiva até o sexto mês de vida ${ }^{(44,45)}$. Spyrides et al ${ }^{(45)}$ citam que o aleitamento materno pode preparar o paladar infantil para os alimentos consumidos pelos familiares, uma vez que a alimentação materna pode alterar o sabor do leite, o que auxiliaria na aceitação de novos alimentos no período do desmame. Rea ${ }^{(46)}$ mostrou aumento de três meses na duração da amamentação entre as brasileiras no período de 1970 a 2002, porém concluiu que a duração mediana ainda era muito baixa, com variações entre as regiões brasileiras.

Uma das dificuldades encontradas ao estudar a amamentação é a falta de padronização do termo, principalmente quando se utiliza o termo aleitamento exclusivo. Estudos randomizados e metanálise evidenciam que o aleitamento materno pode prevenir a obesidade. Tal hipótese é explicada pelo menor conteúdo energético e protéico comparado às fórmulas lácteas, à presença de fatores bioativos no leite materno que podem modular o metabolismo energético e à presença de leptina que modula o apetite, resultando assim em ganho de peso mais fisiológico ${ }^{(47)}$.

Já a metanálise de seis pesquisas mostrou que há pouca evidência da influência do aleitamento materno na mortalidade por doenças cardiovasculares na fase adulta, mas indicou forte evidência de benefícios em relação aos fatores de risco cardiovascular $^{(48)}$.

\section{Transição comportamental}

A inatividade física está aliada à transição nutricional. A urbanização e a industrialização criaram um ambiente com reduzida prática de atividade física, devido à exposição excessiva a televisão, jogos eletrônicos, computadores e serviços de pronta entrega no domicílio.

Estudo mostra que reduzir as horas gastas em frente à televisão diminui o IMC ${ }^{(49)}$. Além disso, observa-se que crianças que permanecem mais tempo assistindo televisão apresentam consumo mais elevado de alimentos com alto valor calórico. Além deste fato, durante os programas infantis, são exibidas propagandas de alimentos hipercalóricos e de baixa densidade nutricional ${ }^{(50)}$. A explicação para estas relações, segundo os pesquisadores, é o fato de que eventos externos ao ato de se alimentar desviam a atenção e diminuem a consistência dos reflexos da saciedade.

Pierine et $a^{(51)}$, ao avaliarem o nível de atividade física de

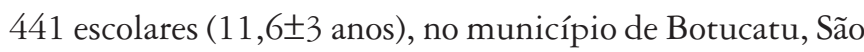
Paulo, verificaram que $54 \%$ dos alunos eram insuficientemente ativos e $6 \%$ sedentários, independentemente do estado nutricional. Maitino ${ }^{(52)}$ verificou que a inatividade física esteve presente em $42 \%$ dos alunos de periferia de Bauru, São Paulo. Em Niterói, Rio de Janeiro, ${ }^{(53)}$ este índice atingiu $85 \%$ dos meninos e $94 \%$ das meninas. Essas diferenças podem ser creditadas, em parte, às diferenças metodológicas entre os estudos ${ }^{(53)}$.

Baruki et al ${ }^{(54)}$ encontraram uma relação entre o estado nutricional da criança e o nível de atividade física, mostrando que crianças eutróficas eram mais ativas, praticavam atividades físicas mais intensas e gastavam menos tempo assistindo à televisão, comparadas às crianças com sobrepeso/ obesidade.

A prática freqüente de exercícios físicos diminui o risco de obesidade, atua na regulação do balanço energético, influencia na distribuição do peso corporal, preserva e mantém a massa magra, além de promover perda de peso corporal ${ }^{(55)}$.

\section{Considerações finais}

A revisão dos dados sugere que as transições demográfica, epidemiológica e nutricional ocorridas nas últimas décadas são os principais fatores para o excesso de peso de crianças e adolescentes, uma vez que estas transições proporcionaram grandes alterações no estilo de vida da sociedade. A inserção da mulher no mercado de trabalho dificultou a prática do aleitamento materno exclusivo até o sexto mês e, posteriormente, o preparo de refeições no domicílio, o que, por sua vez, propiciou o consumo de alimentos industrializados e a ingestão maior de açúcares e gordura. Com a urbanização e o aumento da violência nas grandes cidades, o padrão de vida das crianças e adolescentes se modificou, aumentando o tempo despendido diante de televisores ou computadores. O desmame precoce, a alimentação pouco balanceada e a inatividade física geraram um ambiente propício para o sobrepeso e obesidade. 


\section{Referências bibliográficas}

1. Passmore R, Nicol BM, Rao MN, Beaton GH, Demaeyer EM. Consumo recomendado de energia e nutrientes. In: Organização Mundial de Saúde. Manual das Necessidades Nutricionais Humana. São Paulo: Atheneu; 2003. p.1-5.

2. Formiguera $X$, Cantón A. Obesity: epidemiology and clinical aspects. Best Pract Res Clin Gastroenterol 2004;18:1125-46.

3. Barros Filho AA. Obesity: a puzzling desorder. J Pediatr (Rio J) 2004;80:1-3.

4. Whitaker RC, Wright JA, Pepe MS, Seidel KD, Dietz WH. Predicting obesity in young adulthood from childhood and parental obesity. N Engl J Med 1997;337:869-73.

5. Gillman MW, Rifas-Shiman SL, Frazier AL, Rockett HR, Camargo CA Jr, Field $\mathrm{AE}$ et al. Family dinner and diet quality among older children and adolescents. Arch Fam Med 2000;9:235-40.

6. Killien MG. Women and employment: a decade review. Annu Rev Nurs Res 2001;19:87-123.

7. Zeferino AM, Barros Filho AA, Bettiol H, Barbieri MA. Monitoring growth. J Pediatr (Rio J) 2003;79 (Suppl 1):S23-32.

8. Romani SAM, Lira PIC. Fatores determinantes do crescimento infantil. Rev Bras Saude Matern Infant 2004:4:15-23.

9. Batista Filho M, Rissin A. Nutritional transition in Brazil: geographic and temporal trends. Cad Saude Publica 2003;19 (Suppl 1):S181-191.

10. Barker DJ. Mothers, babies and disease in later life. London: British Medical Journal Books; 1994.

11. World Bank. Repositioning nutrition as central to development - a strategy for large-scale action. Washington: World Bank; 2006.

12. Costa RF, Cintra IP, Fisberg M. Prevalência de sobrepeso e obesidade em escolares da cidade de Santos, SP. Arq Bras Endocrinol Metab 2006;50:60-7.

13. Wang Y, Monteiro C, Popkin BM. Trends of obesity and underweight in older children and adolescents in the United States, Brazil, China, and Russia. Am J Clin Nutr 2002;75:971-7.

14. Brasil - Ministério do Planejamento, Orçamento e Gestão. Instituto Brasileiro de Geografia e Estatística. Pesquisa de Orçamentos Familiares (POF) 2002-2003: antropometria e análise do estado nutricional de crianças e adolescentes no Brasil. Rio de Janeiro: IBGE - Ministério da Saúde - Ministério do Planejamento, Orçamento e Gestão; 2006.

15. Anjos LA, Castro IR, Engstrom EM, Azevedo AM. Growth and nutritional status in a probabilistic sample of schoolchildren from Rio de Janeiro, 1999. Cad Saude Publica 2003;19 (Suppl 1):171-9.

16. Giugliano R, Carneiro EC. Factors associated with obesity in school children. J Pediatr (Rio J) 2004;80:17-22.

17. Soar C, Vasconcelos FAG, Assis MAA, Grosseman S, Luna ME. Prevalência de sobrepeso e obesidade em escolares de uma escola pública de Florianópolis, Santa Catarina. Rev Bras Saude Mater Infant 2004;4:391-7.

18. Ronque ERV, Cyrino ES, Dórea VR, Serassuelo Junior H, Galdi EHG, Arruda $M$. Prevalência de sobrepeso e obesidade em escolares de alto nível socioeconômico em Londrina, Paraná, Brasil. Rev Nutr 2005;18:709-17.

19. Capelli JD, Koifman S. Evaluation of the nutritional status of the Parkatêjê indigenous community in Bom Jesus do Tocantins, Pará, Brazil. Cad Saude Publica 2001;17:433-7.

20. Silva GAP, Balaban G, Motta MEF. Prevalência de sobrepeso e obesidade em crianças e adolescentes de diferentes condições socioeconômicas. Rev Bras Saude Mater Infant 2005;5:53-9.

21. Lamounier JA. Situação da obesidade na adolescência no Brasil. Simpósio sobre obesidade e anemia carencial na adolescência. São Paulo: Instituto Danone; 2002. p. 15-31.

22. Souza MR, Bezerra CS, Mazzariol RA, Leite BPF, Liberatore Junior RDP. Análise da prevalência de resistência insulínica e diabetes mellitus tipo $2 \mathrm{em}$ crianças e adolescentes obesos. Arq Cienc Saude 2004;11:215-8.

23. Baker JL, Olsen LW, Sørensen TI. Childhood body-mass ilndex and the risk of coronary heart disease in adulthood. N Engl J Med 2007;357:2329-37.
24. Frederiksen $\mathrm{H}$. Feedbacks in economic and demographic transition. Science 1969;166:837-47.

25. Laurenti R. Transição demográfica e transição epidemiológica. Abstract do I Congresso Brasileiro de Epidemiologia; 1990 Sept 2-6; Campinas Brasil. p. 143-65.

26. Popkin BM. Nutritional patterns and transitions. Popul Dev Rev 1993;19:138-57.

27. Popkin BM. Global nutrition dynamics: the world is shifting rapidly toward a diet linked with noncommunicable disease. Am J Clin Nutr 2006;84:289-98.

28. Taddei JA, Colugnati FA, Rodrigues EM. Transição nutricional em menores de cinco anos: Evidências dos inquéritos antropométricos brasileiros. In: Cardoso AL, Lopes LA, Taddei JAAC, editores. Tópicos em nutrição pediátrica. São Paulo: Atheneu; 2004. p. 11-43.

29. Brasil - Ministério da Saúde. IBGE [homepage on the Internet]. Estudo nacional de despesa familiar (ENDEF) (1974-75) [Cited 2008 May 5]. Available from: dtr2004.saude.gov.br/nutricao/evento/reuniao_nacional/2005/documentos/ pof_2002_2004.pdf

30. Triches RM, Giugliani ER. Obesity, eating habits and nutritional knowledge among school children. Rev Saude Publica 2005;39:541-7.

31. Mondini L, Levy RB, Saldiva SR, Venâncio SI, de Azevedo Aguiar J, Stefanini ML. Overweight, obesity and associated factors in first grade schoolchildren in a city of the metropolitan region of São Paulo, Brazil. Cad Saude Publica 2007;23:1825-34.

32. Ramos M, Stein LM. Development children's eating behavior. J Pediatr (Rio J) 2000;76 (Suppl 3):S229-37.

33. Philippi ST, Colucci AC, Cruz AT, Ferreira MN, Coutinho RL. Alimentação saudável na infância e na adolescência. In: Curso de atualização em alimentação e nutrição para professores da rede pública de ensino. Piracicaba: Escola Superior de Agricultura Luiz de Queiroz; 2000. p. 46-60.

34. Barbosa RM, Croccia C, Carvalho CG, Franco VC, Salles-Costa R, Soares EA. Food intake by children based on the Brazilian food guide pyramid for young children. Rev Nutr 2005;18:633-41.

35. Isganaitis E, Lustig RH. Fast food, central nervous system insulin resistence, and obesity. Arterioscler Thromb Vasc Biol 2005;25:2451-62.

36. Bray GA, Nielsen SJ, Popkin BM. Consumption of high-fructose corn syrup in beverages may play a role in the epidemic of obesity. Am J Clin Nutr 2004;79:537-43.

37. Cordain L, Eaton SB, Sebastian A, Mann N, Lindeberg S, Watkins BA et al. Origins and evolution of the Western diet: health implications for the $21^{\mathrm{st}}$ century. Am J Clin Nutr. 2005;81:341-54.

38. World Health Organization. WHO Global strategy on diet, physical activity and health: European regional consultation meeting report. Copenhagen: WHO; 2003. p. 5-28.

39. Mensink RP, Katan MB. Effect of dietary trans fatty acids on high-density and low-density lipoprotein cholesterol levels in healthy subjects. N Engl J Med 1990;323:439-45.

40. Capriles V, Arêas JA. Development of snacks with reduced saturated and trans fatty acids contents. Cienc Tecnol Aliment 2005;25:363-9.

41. Kaur H, Hyder ML, Poston WS. Childhood overweight: an expanding problem. Treat Endocrinol 2003;2:375-88.

42. Bray GA, Nielsen SJ, Popkin BM. Consumption of high-fructose corn syrup in beverages may play a role in the epidemic of obesity. Am J Clin Nutr 2004;79:537-43.

43. Kavey RE, Daniels SR, Lauer RM, Atkins DL, Hayman LL, Taubert K. American Heart Association guidelines for primary prevention of atherosclerotic cardiovascular disease beginning in childhood. Circulation 2003;107: $1562-6$.

44. World Health Organization [homepage on the internet]. The optimal duration of exclusive breast feeding. 2001 [cited 2008 May 5]. Available from: http:// www.who.int/inf-pr-2001/en/note2001-07.html

45. Spyrides MHC, Struchiner CJ, Barbosa MTS, Kac G. The effect of breastfeeding practices on infant growth. Rev Bras Saude Mater Infant 2005;5:145-53. 
46. Rea MF. A review of breastfeeding in Brazil and how the country has reached ten months' breastfeeding duration. Cad Saude Publica 2003;19 (Suppl 1):S37-45.

47. American Institute for Cancer Research [homepage on the Internet]. Food, nutrition, physical activity, and the prevention of cancer: a global perspective. [cited 2007 Oct 29]. Available from: http://www.aicr.org/site/ PageServer?pagename=res_rc_home

48. Owen CG, Whincup PH, Odoki K, Gilg JA, Cook DG. Infant feeding and blood cholesterol: a study in adolescents and a systematic review. Pediatrics 2002;110:597-608.

49. Hill JO, Peters JC. Environmental contributions to the obesity epidemic. Science 1998;280:1371-4.

50. Temple JL, Giacomelli AM, Kent KM, Roemmich JN, Epstein LH. Television watching increases motivated responding for food and energy intake in children. Am J Clin Nutr 2007;85:355-61.
51. Pierine DT, Carrascosa AP, Fornazari AC, Watanabe MT, Catalani MC, Fukuju MM et al. Composição corporal, atividade física e consumo alimentar de alunos do ensino fundamental e médio. Motriz 2006;12:113-24.

52. Maitino EM. Aspectos de risco coronariano em casuística de crianças de escola pública de primeiro grau em Bauru, SP. Rev Brasil At Fis Saude 1997;2: 37-52.

53. da Silva RC, Malina RM. Level of physical activity in adolescents from Niterói, Rio de Janeiro, Brazil. Cad Saude Publica 2000;16:1091-7.

54. Baruki SBS, Rosado LEFP, Rosado GP, Ribeiro RCL. Associação entre estado nutricional e atividade física em escolares da Rede Municipal de Ensino em Corumbá-MS. Rev Bras Med Esporte 2006;12:90-4.

55. Gillis LJ, Kennedy LC, Bar-Or O. Overweight children reduce their activity levels earlier in life than healthy weight children. Clin J Sport Med 2006;16: 51-5. 